(C) 2006 IEEE. Personal use of this material is permitted. Permission from IEEE must be obtained for all other uses, in any current or future media, including reprinting/republishing this material for advertising or promotional purposes, creating new collective works, for resale or redistribution to servers or lists, or reuse of any copyrighted component of this work in other works. 


\title{
Dynamic Web-Based Tutorial Tool
}

\author{
Benedykt S. Rodanski
}

\begin{abstract}
As the number of students increases quite dramatically in almost every subject taught at a university, delivering tutorials to large classes and providing timely assessment and feedback becomes the major challenge. These problems are especially acute in technical field-of-practice subjects that aim to teach specific numeracy and problem solving skills. In this paper we describe a generic framework for delivering and assessing tutorials over the Web that would enable us to create Web tutorials in several technical subjects. A sample implementation in one of the early stage technical subjects (Electronics \& Circuits) will be described. The system, implemented in ASP .NET, presents each student with a number of unique sets of problems (generated by software, not randomly chosen from a fixed pool), guides him/her through the solutions and grades the work. A strict deadline is enforced for each set to encourage systematic approach to learning.
\end{abstract}

Index Terms-educational technology, Web-based education, dynamic tutorials, client-server software.

\section{INTRODUCTION}

$\mathbf{I}_{\mathrm{d}}^{\mathrm{N}}$ recent years the number of students increased quite dramatically in most subjects taught at universities. Not surprisingly, with such an increase, the overall quality of student intake dropped significantly. To quote a former Australian federal education minister: "The funding system forces universities to fill every place, no matter how low the student standard." (Fin. Rev., 04/04/2004). Large classes and low entrance barriers do little to increase student motivation. In this difficult environment, delivering tutorials and providing timely assessment and feedback became the major challenge.

These problems are especially acute in early stage technical field-of-practice subjects that aim to teach specific numeracy and engineering problem solving skills. These skills can only be acquired (and retained) by extensive and systematic practice. Every engineering textbook has a large number of worked examples, exercises and end of chapter problems to be solved by students. The textbook authors hope, of course, that students will devote considerable time and effort to solving these problems.

The challenge is, however, how to motivate the students to focused and systematic learning. A weekly quiz, run during every tutorial session, or several written assignments during a semester are not a feasible options any more due mainly to large volume of marking involved.

The widespread application of WWW technology in education resulted in a variety of methods of courseware

B.S. Rodanski is with Faculty of Engineering, University of Technology, Sydney (UTS), P.O. Box 123, Broadway, NSW 2007, Australia (ben.rodanski@uts.edu.au) delivery over the Web. The most common is a static Web content such as subject outline, course notes and other textbased material, because it is the easiest to create. However, interactive content, where the Web application collects and processes input from the user and then gives immediate feedback, is generally far more pedagogically engaging [1]. Web-based activities that are assessed interactively are very good at motivating participation (as long as marks can be gained; the power of instantaneous feedback alone should not be overestimated). Traditional teaching methods, like tutorial class sessions and written assignments, have lost their motivational advantage mainly because the difficulties in providing timely feedback. Plagiarism in written assignments is also a growing concern.

The proposed generic framework for delivering and assessing tutorials over the Web would enable us to create Web tutorials in many technical subjects. Such system presents each student with a number of unique sets of problems, guides him/her through the (multipart) solutions and grades the work. A strict deadline is enforced for each set to encourage systematic approach to learning. A modification of the system is also used as a very effective examination tool.

Several Web-based tutorial systems have been developed for engineering subjects (e.g. [2], [3]), but to my knowledge, none of them implements the concept of generating unique questions by software. The variety is achieved there by randomly selecting from a large pool of static questions.

\section{THE WEB TUTOR FRAMEWORK}

The major goal of this project was to create a system that would encourage engineering students to systematic learning. In my experience, providing feedback - no matter how timely and how detailed - is not enough to motivate students to practice problem solving; it is always the marks they receive for such activities that motivate them.

With small classes it is possible to conduct a weekly quiz that is marked by a tutor. For large classes this resourceintensive method is no longer feasible.

Another popular solution is an on-line quiz. Commercially available systems, like Blackboard or WebCT, include tools for setting up and conducting such quizzes. These tools, however, have not been designed for engineering problem solving exercises. The two major drawbacks are: First, only character string answers are permitted (e.g., answers "1", " $1.0 "$, " $1 \mathrm{e} 0$ ", etc., although representing the same numerical value, will be treated as different; also, there is no mechanism for assessing the accuracy of the answer). Second, if we want to introduce some variation, all questions and answers must be prepared in advance and stored in a pool from which they are picked at random. 


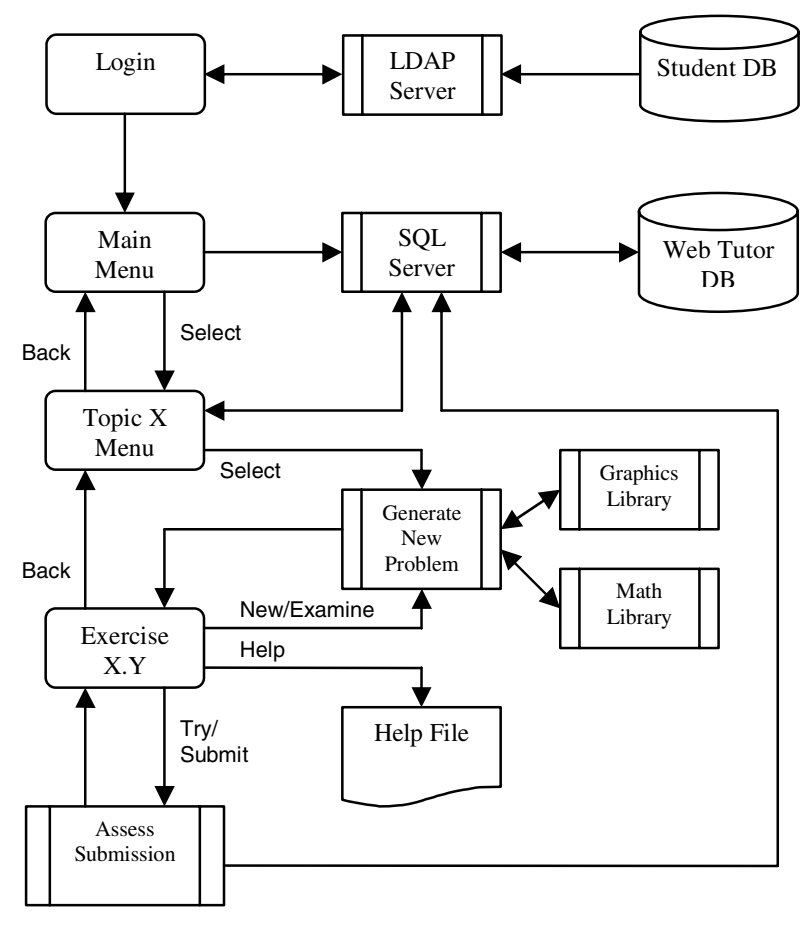

Fig. 1. Web Tutor - simplified data flow.

A much more interesting and effective technique is to generate a unique question each time the exercise is attempted. Questions should vary not only in numerical values of parameters, but also in topology. Such an approach would also facilitate learning, not just testing the knowledge, as students could attempt similar problems an unlimited number of times, never seeing the same problem twice. When they indicate their readiness to be examined, a new (but similar) problem is generated.

Numerical answers in all possible formats should be accepted and the grading of the answer may depend on its accuracy.

The basic block diagram of the Web Tutor framework is shown in Fig. 1. Since the system is to be used for assessment, the users must be authenticated. This is facilitated by a login process that uses a secure LDAP server and a database with all valid user names and passwords.

Once the user is authenticated, he/she is presented with a Main Menu screen from which a particular topic is selected. A Topic Menu is then displayed and the user selects an Exercise from a list.

At this point the system generates a unique problem and presents it to the user. Problem formulation involves generating random parameters, solving the problem (to make sure that it makes sense as well as to provide the correct answers for grading and feedback) and drawing a picture.

User then solves the problem, enters his/her answers and compares them with the correct solution. A trial grading (not recorded for assessment purpose) is also provided. The process of generating new problems and giving feedback on user's solutions may be repeated unlimited number of times.

Help (in the form of either static or interactive Web content) is also available during the trials.
Eventually, the user indicates his/her readiness to be examined on the material studied. A new problem is generated and user's answer is assessed. The test can only be taken once and the answer cannot be corrected later.

Not shown on the diagram in Fig. 1 are the utility programmes that are necessary in a practical implementation of the Web Tutor framework. One of those creates the marks spreadsheet for the class. The other utility allows viewing of any submission by a particular student. The latter utility is very useful in resolving disputes with students as well as in debugging the software.

\section{SAMPLE IMPLEMENTATION}

The framework, described above, was implemented in the undergraduate subject Electronics \& Circuits, taught at the Faculty of Engineering, UTS. The software, called E\&C Web Tutor, was written as an ASP .NET client-server application with the 'code behind' in VB .NET.

\section{A. The Web Tutor}

There are 12 topics, linked to weekly lectures and closely following the subject material. Each topic contains 5-8 exercises. To illustrate the concept of generating random problems, we will present here one of the 65 exercises in the system.

The exercise is designed to teach students how to analyse electrical circuits containing resistors and current sources. A student who selects this exercise will be initially presented with a screen similar to the one shown in Fig. 2. To help students learn the solution process, a set of equations describing the circuit can be displayed (by pressing the "Show Equations" button). Both symbolic and numeric forms of the circuit equations are generated concurrently with the circuit diagram. The result is shown in Fig. 3.

\section{Electronics \& Circuits: Web Tutor}

Topic 2: Resistive Circuits

Exercise 2.3: Node Voltage Analysis; Circuits with I and R

Calculate the node voltages in a circuit shown below.

To test your solution (without recording the score) enter your answers in the text boxes and click on the Try button. You may try as many times as you want.

When you are ready to take the test, click on the Examine button. You will be presented with a new problem. Your answer and score will be recorded for assessment purpose.

To get a perfect score (100), the accuracy of your answer(s) must be $1 \%$ or better.

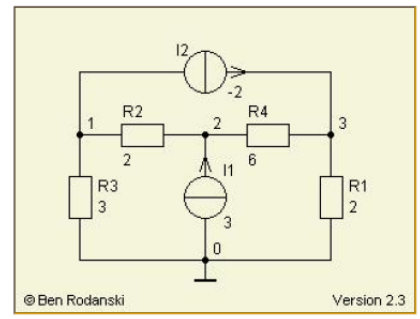

Examine

\section{New Circuit}

Equation Solver

Your solution

$\mathrm{v} 1=$

$\mathrm{v} 2=$

v3 $=$

$$
\text { Try }
$$

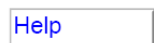

Help
Fig. 2. A sample screen for Exercise 2.3. 
Node Voltage Equation $[\mathbf{G}]^{*}[\mathbf{v}]=[\mathbf{i}]$ :

Symbolic:

\begin{tabular}{l|l|l|l|l|}
\hline$G 2+G 3$ & $-G 2$ & & $v 1$ & -12 \\
\hline$-G 2$ & $G 2+G 4$ & $-G 4$ & $*$ & $v 2=$ \\
& $-G 4$ & $G 1+G 4$ & v3 & 12 \\
& &
\end{tabular}

\section{Numeric:}

\begin{tabular}{|c|c|c|c|}
\hline 833333 & -0.500000 & 0.000000 & 2.0 \\
\hline 50 & 0.666667 & -0.166667 & * V2 $=3.0$ \\
\hline 00000 & -0.166667 & 0.666667 & v3 \\
\hline
\end{tabular}

Fig. 3. Fragment of a screen after pressing the "Show Equations" button for the circuit in Fig. 2.

The number of possible unique topologies within a given graph structure is 15; each component is randomly assigned one of 50 different values. This brings the number of unique problems to $15 * 50^{6}>23 * 10^{11}$. Every time the "New Circuit" button is pressed, another random permutation of four resistors and two current sources is generated and each element is assigned a random value; one possible result is shown in Fig. 4. A new circuit is also generated when the user requests to be examined.

When the user enters his/her solution and presses the "Try" button, the true solution and the trial score are given (Fig. 5).

In the exam mode all but the "Equation Solver" button disappear and the "Examine" button is replaced by the "Submit" button. Once the submission is made, it is graded, recorded in the data base and the grade is given to the user. If the user returns later to the problem, the "Examine" button will be disabled, so the exam can be taken only once. The "Examine" button is also disabled when the problem is viewed after the submission deadline.

\section{B. The Web Exam}

Several of the Web Tutor Exercises are incorporated into another Web application: the $E \& C W e b$ Exam. It is used to conduct a (formal) final examination in the subject. The exam is run in the controlled environment where student collaboration, use of outside help, unauthorised software, etc., is not possible. All students are given problems of the same type, but differing in both topology and numerical parameters, as in the Web Tutor exercises. The general data flow of the Web Exam is similar to the one in Fig. 1, with three major differences.

Firstly, in the Web Exam there is no "New Circuit" command; once issued, the exam question is recorded in the database and cannot be changed. However, at any time during the exam students can return to a submitted question to review and/or change the results.

Secondly, the "Help" command as well as other help features (e.g. the "Show Equations" command) are no longer available. Access to the Web Tutor's help files is also disabled. Students are allowed to bring a one page, hand written 'aide memoir.'

Finally, there is no immediate feedback. Neither the correct results nor the score is given to the student who submits the

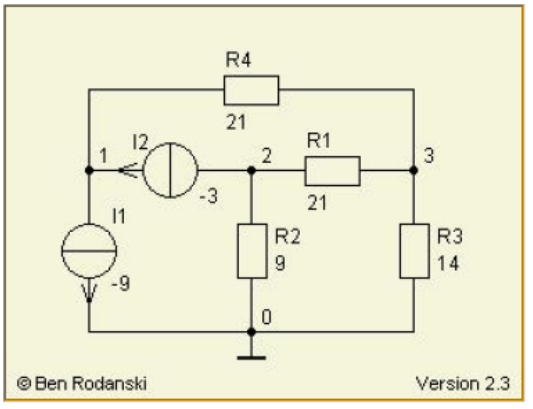

New Circuit

Equation Solver

Your solution:

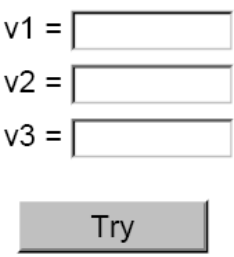

Fig. 4. An example of a different circuit, generated within the same frame.

\section{Electronics \& Circuits: Web Tutor}

Topic 2: Resistive Circuits

Exercise 2.3: Node Voltage Analysis; Circuits with I and R

\section{Calculate the node voltages in a circuit shown below.}

Try command issued.

The exact answers are displayed next to your entries.

Your score: 90 (High Distinction).

Your score is not recorded for assessment purpose.

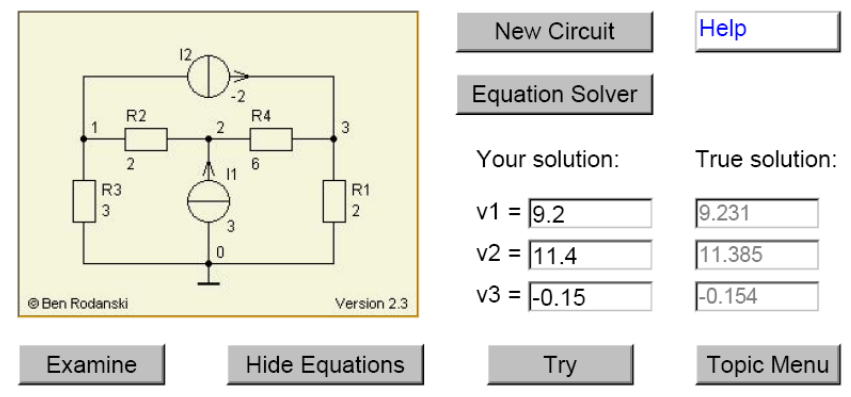

Fig. 5. Result of a successful try for the circuit in Fig. 2.

answer. This prevents undue anxiety during the exam. Interested students can view their exam questions and answers after the exam (surprisingly few does).

\section{RESULTS}

\section{A. Pre-Web Tutor Situation}

In the last 9 semesters prior to the introduction of the Web Tutor the subject was taught using consecutive editions of the same textbook [2] and the same assessment technique. There were no formal tutorials, but tutors were available for consultations at various times during the week. Students were given a set of 'compulsory' problems (from the textbook) to be solved each week and recorded in a Journal. The assessment consisted of: a mid semester exam (30\%), laboratory (15\%) and the final exam (55\%). Questions in both exams were randomly chosen from the 'compulsory' set (the fact well advertised to students). Despite the fact that the exam questions were fairy predictable, the results were very poor, as can be seen from the graph in Fig. 6. The mean value of the 


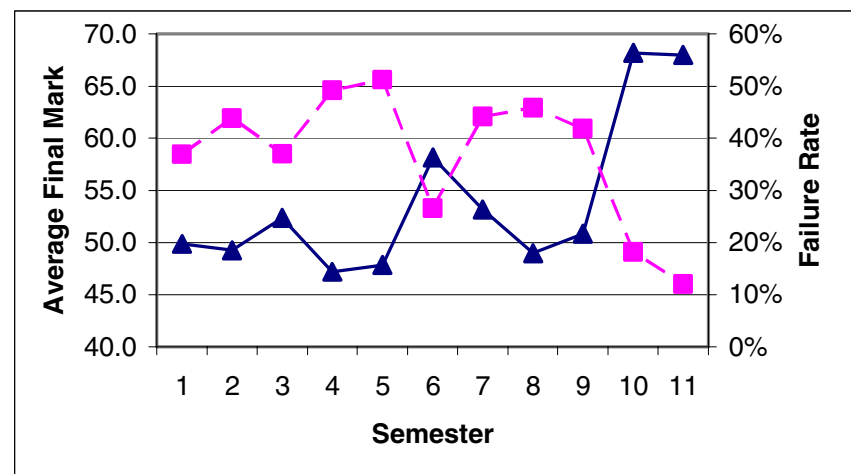

Fig. 6. Assessment results pre- and post-Web Tutor ( $\mathbf{-}$ - failure rate,

$\boldsymbol{\Delta}$ - average final mark).

average final mark for 9 previous semesters was $51 \%$; the mean value of the failure rate in the same period was $42 \%$.

\section{B. Results after introduction of the Web Tutor}

The E\&C Web Tutor was fully implemented in 2005 with very encouraging results. The average Web Tutor result was $75 \%$ and the average final mark was $68 \%$ in both semesters. The failure rate dropped well below $20 \%$. This dramatic improvement can be attributed to the fact that students were motivated (by the marks given for timely and correct submission of their solutions) to practice on 65 exercises, covering the entire subject. The final exam was a supervised formal exam, conducted using a modification of the Web Tutor, called the E\&C Web Exam. As in the past (traditional) exams, students knew that the exam questions will be very similar to the tutorial problems.

\section{Student feedback}

Feedback from students on using the new approach to tutorials is very good. I have received a large number of unsolicited opinions, mostly positive. The majority of students think the Web Tutor helps them learn the material much better than a traditional approach. They value its availability 'anywhere, anytime' and the fact that it forces them to focus on particular topic every week, yet allowing for unlimited trials. I also receive many enquiries as to when similar software will be introduced in other subjects. The following is a typical student comment:

"[...] I would like to back the comments on the discussion board about Web Tutor. I think it is a fantastic learning aid. Not only does it force the student to do the problems, and learn, but also provides limitless problems on exactly what we need to know. Thanks again for Web Tutor."

\section{Common problems in using Web Tutor}

Apart from the few inevitable software 'bugs' that were detected and corrected early, there are some common problems, reported by students. One problem is related to the fact that the Web Tutor can only be correctly navigated with its internal commands. Students, using the browser's navigation commands ("Forward" and "Back"), as well as trying to open more than one Web Tutor window at the time, run into difficulties and sometime unwittingly submit an incorrect or incomplete answer. The software warns users against these practices at every possible occasion, yet many students simply ignore the warnings. These problems occur mainly in the first few weeks of the semester, but initially create a heavy e-mail traffic and a large number of comments on the subject discussion board.

Another common problem is caused by students making 'silly' mistakes, mainly due to carelessness, in either calculations or entering the results. A typical example is entering the wrong sign of the exponent (e.g. 1E-3 instead of $1 \mathrm{E}+3$ ) or an incorrect unit prefix [confusing ' $\mathrm{M}$ ' (for mega) with ' $\mathrm{m}$ ' (for milli) is the most frequent 'silly' mistake]. In the near future the Web Tutor software will be made more 'intelligent' to deal at least with some more obvious of these cases. Ultimately, however, students must learn that in engineering calculations the accuracy of the final result is as important as the correctness of the process they follow to obtain that result.

\section{CONCLUSION}

In teaching technical, field-of-practice engineering subjects, the traditional approach of lecture + class tutorial + lab is no longer effective. Class tutorials can be effectively replaced by Web-based activities.

A general framework of a Web-based tutorial delivery and assessment system was presented. The framework was implemented in one of the early stage subjects in the Electrical/Computer Systems/Telecomm Engineering course at the Faculty of Engineering, UTS. The application, called the E\&C Web Tutor, is an ASP .NET client-server software, written in VB .NET. Interested readers are welcome to access the software at: http://services.eng.uts.edu.au/ ecwebtutor/guest_login.aspx

The initial results are excellent: average final mark in the subject rose from 51 to 68 , and the failure rate dropped from $42 \%$ to $15 \%$. It is still too early to draw any definitive conclusions, but we hope that future results will confirm the validity of our approach.

Further work will concentrate on finding out how the Web Tutor influences the results in subsequent subjects, especially the ones for which $\mathrm{E} \& \mathrm{C}$ is the prerequisite.

\section{ACKNOWLEDGEMENT}

The successful implementation of the Web Tutor would not be possible without generous help from the Faculty Web Development team, lead by Michel de la Villefromoy: Rodrigo del Busto and Frank Ng. Their involvement in the project is greatly appreciated.

\section{REFERENCES}

[1] C. Bennett, Multipart Homework Problems over the Web. Computing in Science \& Engineering, 1, 2000, pp. 87-91,95.

[2] N. Scott and B. Stone, A Flexible Web-based Tutorial System for Engineering, Maths and Science Subjects. Global J. of Eng. Educ., Vol. 2, No. 1, 1998, pp. 7-16.

[3] S. Hussmann and C. Smail, The Use of Web-based Learning and Communication Tools in Electrical Engineering. Australasian J. of Eng. Educ., online publication 2003-01. 